\title{
Modelagem para localização de hubs no transporte de encomendas expressas
}

\author{
Javier Antonio Timaná Alamo \\ Marco Antonio Brinati
}

POLI-USP

\section{Resumo}

Na presente pesquisa se propõe um modelo matemático de programação linear com variáveis binárias 0/1, para projetar a configuração de uma rede de distribuição de encomendas expressas, visando minimizar os custos e garantindo um bom nível de serviço. O modelo, que é uma modificação da formulação proposta por O’Kelly, define as posições dos hubs, a alocação deles às demais instalações físicas e a construção de roteiros com apenas uma parada intermediária, o que confere mais agilidade ao atendimento da demanda de transporte de carga. Considera-se a instalação de um único hub maior (Hub Principaß), que serve como ponto de transbordo para os fluxos de carga entre as distintas regiões de um território, e um conjunto de terminais regionais (mini-hubs), servindo como ponto de conexão, unicamente, para os fluxos de carga existentes em um determinado raio de cobertura. Foram propostas extensões que incluem uma restrição da capacidade operacional do Hub Principal, para evitar seu congestionamento, e duas diferentes estratégias de induzir o aumento de fluxo de carga manipulado pelos mini-hubs. 0 modelo proposto e suas extensões foram aplicados ao estudo de um caso real, obtendo-se resultados consistentes e uma redução significativa no custo total da rede de distribuição da empresa analisada.

\section{Palavras-chave}

Localização industrial, pesquisa operacional, terminais de carga, transporte de carga.

\section{Modelling for location hubs in the express cargo transport}

\begin{abstract}
This study proposes a mathematical model of linear programming with binary variables $0 / 1$ to project the configuration of a distribution network for express cargo which aims to minimize costs and guarantee a high level of service. The model, which is a modification of the formulation proposed by O'Kelly, defines the position of the hubs, their allocation regarding the other physical installations, and the building of itineraries with only one intermediate stop, which increases agility in meeting the demand of cargo transport. It assumes the installation of a single, major hub (Main Hub), which serves as the transfer point for the flow of cargo between distinct regions of a territory and a set of regional terminals (mini hubs) serving as connection points solely for the flow of cargos existing within a determined area of coverage. Extensions were proposed with capacity constrains for the Main Hub, thereby avoiding congestion, and two different strategies to induce an increase of the flow of cargo manipulated by the mini-hubs. The proposed model and its extensions were applied to an actual case study, obtaining consistent results and a significant reduction in the total cost for the distribution network of the company studied.
\end{abstract}

Key words

Industrial localization, operational research, cargo terminals, cargo transport. 


\section{INTRODUĈ̣̃O}

O presente trabalho trata do problema de localizaçãoalocação de hubs para uma empresa de transporte de encomendas expressas.

A carga transportada pelo serviço de encomendas expressas abrange documentos, amostras, catálogos, produtos vendidos pela Internet, peças de reposição e pacotes de pequeno volume a serem entregues em um prazo de tempo relativamente curto, entre duas localidades distantes entre si, especificadas pelo cliente.

Esse tipo de serviço de transporte engloba as operações de coleta na origem, transferência da carga até terminais intermediários, consolidação (criação de grandes carregamentos a partir de vários outros pequenos), classificação, desconsolidação (formação de carregamentos menores a partir de um volume maior) e a entrega no destino, sendo o tipo de serviço mais comumente oferecido o de tipo porta-a-porta.

A rede de distribuição física do transporte de encomendas expressas se caracteriza por ser de tipo hub-and-spoke, formada por terminais de consolidação $(h u b s)$ e instalações físicas (lojas, agências, centros operacionais) alocadas a cada $h u b$. Nesse tipo de configuração de rede, de múltiplas origens e destinos, os hubs servem como pontos de transbordo ou de conexão para o fluxo de carga entre duas instalações, permitindo, dessa maneira, substituir uma grande quantidade de conexões diretas entre todos os nós de uma rede por uma menor quantidade de conexões indiretas.

Em uma rede hub-and-spoke, a demanda de transporte entre um nó origem e um nó destino tem que ser movimentada ao longo de uma rota que atravessa pelo menos um hub. Conseqüentemente, a aglomeração de carga nos hubs permite alcançar economias de escala, reduzindo consideravelmente o custo operacional da rede.

É de destacar que, pelo dinamismo exigido pelo mercado, os usuários do sistema de carga expressa tornaram-se cada vez mais exigentes em relação a um serviço que seja barato, rápido, seguro e que cumpra os prazos de entrega estabelecidos.

Por esse motivo, os sistemas de entrega de carga expressa trabalham com o conceito de que o tempo de entrega oferecido ao cliente (geralmente dentro de um ou dois dias) tem que ser garantido; por conseguinte, as restrições de tempo são de interesse primordial no momento de projetar sua rede de distribuição. Conseqüentemente, a principal preocupação de muitas empresas do setor é encontrar uma configuração de distribuição física que leve em conta uma diminuição do número de paradas nos terminais de carga ou evite múltiplas tarefas de classificação que acarretam um consumo de tempo.

Uma ampla revisão da literatura permitiu constatar que o problema de localização de hubs para carga expressa, tal- vez devido a sua complexidade, tem sido objeto de pouca análise (encontrou-se um número bastante limitado de artigos científicos abordando o tema). A maioria das pesquisas realizadas até o momento sobre localização de $h u b s$ foi proposta para projetar redes de transporte aéreo de passageiros, visando unicamente a minimização dos custos variáveis de transporte.

Desta maneira, o presente trabalho pretende formular um modelo matemático que aborde simultaneamente a localização das instalações físicas concentradoras ( $h u b s$ ), a designação das demais instalações físicas aos $h u b s$ e a construção de roteiros com um número mínimo de paradas intermediárias. $\mathrm{O}$ tratamento integrado destes fatores, bem como a incorporação de custos mais abrangentes, permitirá ao modelo projetar uma rede de distribuição de mínimo custo e com um bom nível de serviço.

\section{DEFINIC̣ÃO DO PROBLEMA}

O desenvolvimento do presente trabalho pretende resolver o problema de localização-alocação de hubs para um sistema de transporte de encomendas expressas, envolvendo as seguintes decisões:

- A partir de um conjunto de locais candidatos, determinar quantas e quais dessas instalações logísticas deverão assumir o papel de hub.

- Quais das instalações físicas restantes serão alocadas a cada $h u b$ com o objetivo de obter uma rede de distribuição de mínimo custo e um bom nível de serviço?

- Qual será o roteiro utilizado para movimentar as encomendas expressas entre cada par origem-destino? Isto é, por quais e quantos hubs deve passar a demanda de carga para chegar a seu destino?

- Examinar a possibilidade de projetar uma rede de distribuição física com um número mínimo de paradas nos terminais de consolidação.

\section{REVISÃO BIBLIOGRÁFICA}

Existem dois tipos básicos de redes hub-and-spoke, diferenciando-se na maneira como estão conectados os nós secundários (spokes) aos nós principais (hubs).

No primeiro tipo de rede, denominado de Alocação Única (Single Allocation), cada nó secundário está conectado a um único hub, de modo que todo o fluxo que sai ou chega ao nó tem que passar sempre pelo mesmo hub a ele alocado. No segundo tipo de rede, denominado de Alocação Múltipla (Multiple Allocation), cada nó secundário está conectado a mais de um $h u b$, incrementado-se dessa maneira o número das ligações na rede.

A partir das revisões bibliográficas feitas por Campbell (1994), O’Kelly e Miller (1994) e Bryan e O’Kelly (1999), 
foi possível organizar o crescente número de pesquisas científicas desenvolvidas ao longo destes últimos anos sobre a modelagem de localização-alocação discreta de hubs, possibilitando sua classificação em sete grupos, de acordo com suas características e aplicações, tal como mostra a Tabela 1.

O primeiro grupo de formulações engloba os modelos não capacitados que possuem algumas das seguintes características: não impõem limites quanto à quantidade de fluxo que está sendo transferida dos nós secundários aos hubs, não restringem a quantidade de nós secundários a serem alocados aos hubs, nem consideram restrições de capacidade das instalações físicas no problema.
O segundo grupo de modelos abrange formulações matemáticas que impõem restrições sobre a quantidade de carga movimentada pelas instalações físicas. Ou seja, considera redes de distribuição com hubs de capacidade finita.

No terceiro grupo, o modelo Flow Loc tem o atributo de refletir melhor a realidade dos ganhos de escala obtidos pela consolidação do fluxo nos hubs, ao considerar que o fator de redução (economia de escala) que influi sobre o custo de transporte entre dois hubs é variável e está em função do fluxo de carga entre esses dois pontos.

O quarto grupo de modelos, Hub Center, se caracteriza por focalizar sua atenção no tempo de atendimento, estabe-

Tabela 1: Classificação dos modelos de localização discreta de hubs.

\begin{tabular}{|c|c|c|c|c|}
\hline $\begin{array}{l}\text { CLASSE } \\
\text { MODELOS }\end{array}$ & VERSÃO & AUTOR & APLICAÇÃOO & FOCO \\
\hline \multirow{7}{*}{$\begin{array}{l}\text { Localização } \\
\text { de hubs não } \\
\text { capacitados } \\
\text { (p-Hub } \\
\text { Median } \\
\text { Problem] }\end{array}$} & \multirow{4}{*}{ Alocação única } & O’Kelly (1987) & \multirow{10}{*}{$\begin{array}{l}\text { Localização } \\
\text { de terminais } \\
\text { para } \\
\text { transporte } \\
\text { de } \\
\text { passageiros } \\
\text { e carga }\end{array}$} & \multirow{10}{*}{$\begin{array}{l}\text { Custo } \\
\text { total da } \\
\text { rede }\end{array}$} \\
\hline & & Campbell (1994) & & \\
\hline & & Skorin-Kapov D., Skorin-Kapov J. e O’Kelly (1996) & & \\
\hline & & Ernst e Krishnamoorthy (1996) & & \\
\hline & \multirow{3}{*}{ Alocação múltipla } & Campbell (1994) & & \\
\hline & & Skorin-Kapov D., Skorin-Kapov J. e O’Kelly (1996) & & \\
\hline & & Ernst e Krishnamoorthy (1998) & & \\
\hline \multirow{2}{*}{$\begin{array}{l}\text { Localização } \\
\text { de hubs } \\
\text { capacitados }\end{array}$} & Alocação única & Ernst e Krishnamoorthy (1999) & & \\
\hline & Alocação múltipla & Ebery, Krishnamoorthy,Ernest e Boland (2000) & & \\
\hline Flow Loc & Alocação múltipla & O’Kelly e Bryan (1998) & & \\
\hline \multirow{2}{*}{ Hub Center } & & Campbell (1994) & \multirow{4}{*}{$\begin{array}{l}\text { Localização } \\
\text { de bases de } \\
\text { serviço de } \\
\text { emergência }\end{array}$} & \multirow{4}{*}{$\begin{array}{l}\text { Tempo } \\
\text { de } \\
\text { atendimento }\end{array}$} \\
\hline & & Kara e Tansel (2000) & & \\
\hline \multirow{2}{*}{ Hub Covering } & & Campbell (1994) & & \\
\hline & & Kara e Tansel (1999) & & \\
\hline \multirow{9}{*}{$\begin{array}{l}\text { Posições } \\
\text { fixas dos } \\
\text { hubs }\end{array}$} & & Jeng (1987) & \multirow{9}{*}{$\begin{array}{l}\text { Transporte } \\
\text { aéreo de } \\
\text { passageiros } \\
\text { e carga }\end{array}$} & \multirow{9}{*}{$\begin{array}{l}\text { Custo } \\
\text { total } \\
\text { da } \\
\text { rede }\end{array}$} \\
\hline & Lonexoes diretas & Miller (1990) & & \\
\hline & & Flynn e Ratick (1988) & & \\
\hline & $\begin{array}{l}\text { Stopovers } \\
\text { e feeders }\end{array}$ & Kuby e Gray (1993) & & \\
\hline & \multirow{2}{*}{$\begin{array}{l}\text { Maximizar } \\
\text { lucros }\end{array}$} & Daskin e Panayotopoulos (1989) & & \\
\hline & & Dobson e Lederer (1993) & & \\
\hline & congestionamento & Grove e O’Kelly (1986) & & \\
\hline & hubs principal & Hall (1989) & & \\
\hline & e mini-hubs & O’Kelly e Lao (1991) & & \\
\hline \multirow{2}{*}{$\begin{array}{l}\text { Hubs } \\
\text { hierárquicos }\end{array}$} & Irrestrito & O’Kelly (1998) & \multirow{2}{*}{$\begin{array}{l}\text { Carga } \\
\text { expressa }\end{array}$} & \multirow{2}{*}{$\begin{array}{l}\text { Custo rede } \\
+ \text { tempo } \\
\text { entrega }\end{array}$} \\
\hline & $\begin{array}{l}\text { Membros do clube } \\
\text { de embarque }\end{array}$ & O’Kelly (1998) & & \\
\hline
\end{tabular}

Fonte: elaboração dos próprios autores 
lecendo uma função objetivo que visa minimizar o máximo tempo de viagem por uma rota que sai de um nó origem, passa por, pelo menos, um hub e chega a um nó destino.

O quinto grupo de modelos, Hub Covering, se rege pelo princípio de que as demandas dos diversos pontos da rede são cobertas, se as instalações que funcionam como hubs forem localizadas suficientemente próximas dos pontos da rede de modo a atendê-los de acordo com os níveis de serviço pré-especificados.

O sexto grupo de modelos com posições fixas de hubs envolve formulações em que as posições dos hubs são predefinidas de antemão, acrescentando-se diversas extensões que influem sobre a etapa de alocação. Entre as variantes agregadas aparecem: o uso de conexões diretas entre nós secundários, o projeto de redes em que os lucros devem ser maximizados, a presença de congestionamento nos hubs e a adaptação do problema de localização para sistemas de carga expressa.

O sétimo grupo que contempla a implantação de $h u b s$ hierárquicos é uma variante da configuração de uma rede pura hub-and-spoke com aplicação em redes de entrega expressa de pacotes. A modelagem propõe a instalação de um único Hub Principal para o fluxo interregional e a instalação de um conjunto de $h u b s$ de menor porte, denominados mini-hubs, para fluxos regionais.

A revisão bibliográfica abrangente permitiu constatar que os modelos clássicos de localização-alocação para redes hub-and-spoke não são apropriados para o projeto de redes de encomendas expressas. Assim, verificou-se que a maioria deles foi criada para ser aplicada ao transporte aéreo de passageiros, no qual seus usuários são obrigados a fazer viagens com até duas escalas para chegar ao destino final. Entretanto, para os sistemas de transporte de carga expressa é de interesse primordial o tempo total de transferência da carga.

Outra limitação dos modelos clássicos é que a função objetivo só contabiliza os custos variáveis de transporte, enquanto que, para o projeto de redes de encomendas expressas, também são relevantes os custos fixos de operação dos terminais de consolidação, pois o trade-off entre esses dois tipos de custos é responsável, juntamente com o conjunto de restrições do modelo, por controlar o número de terminais de consolidação a serem implantados.

Analisando os modelos encontrados na literatura, conclui-se que o Modelo Irrestrito proposto por O'Kelly (1998) é a formulação matemática que proporciona a configuração de rede mais próxima das características desejadas para o serviço de transporte de encomendas expressas.

A escolha do modelo de O'Kelly, como ponto de referencia, decorre de sua preocupação em elevar o nível de serviço, ao propor uma configuração de distribuição física com roteiros de uma única parada intermediária, e da sua sugestão para a implantação de hubs com diferentes áreas de cobertura, o que confere mais agilidade ao atendimento da demanda de transporte de carga.

\section{MODELAGEM MATEMÁTICA DO PROBLEMA}

A formulação matemática proposta neste trabalho surge de um aperfeiçoamento no modelo de O'Kelly, já que no momento de ser submetida esta formulação a uma bateria de testes computacionais foi possível verificar suas limitações.

\section{principal preocupação de muitas empresas do setor é encontrar uma figuração de distribuição física que em conta uma diminuição do número de paradas nos terminais de carga ou evite múltiplas tarefas de classificação, que implicam um consumo de tempo.}

A seguir, são descritas as modificações introduzidas no modelo de O'Kelly, a fim de propor um novo modelo de programação linear com variáveis binárias $0 / 1$ que solucione o problema de localização-alocação de hubs para um sistema de transporte de encomendas expressas:

- Para a definição da função objetivo, foram adicionados aos custos variáveis de transporte os custos fixos de operação dos terminais de consolidação de carga;

- O parâmetro $p$ que define o número de terminais regionais (mini-hubs) a serem implantados não foi especificado $a$ priori. Assim, quando o modelo foi aplicado a um caso real, foi fixado um limite superior para $p$, de maneira que se torne uma restrição fraca, ficando o cálculo do número exato de mini-hubs por conta de outras restrições do modelo e do balanceamento entre os custos variáveis de transporte e os custos fixos de operação dos terminais de carga;

- Foi incluída no modelo uma restrição que traduz a idéia original de O'Kelly de restringir o raio de cobertura dos terminais regionais;

- Quando foi testado o modelo de O'Kelly, constatou-se que alguns nós da rede resultavam alocados a mais de um mini-hub, conduzindo, eventualmente, a linhas na rede com baixo fluxo de carga, o que implicaria uma baixa taxa de aproveitamento dos veículos ou uma redução das 
freqüências das viagens. Com a finalidade de superar essa limitação do modelo de O'Kelly, foi acrescentada uma restrição que estabelece que cada local deve estar conectado a um único mini-hub para trocas regionais de carga.

A configuração da rede de distribuição física obtida com o modelo proposto, ilustrada na Figura 1, tem as seguintes características:

- Considera uma rede hub-and-spoke de alocação múltipla em que cada nó está conectado a um Hub Principal, para interagir com os nós das restantes regiões de um território, e ao mesmo tempo, conectado a um mini-hub, para interagir com os nós da sua própria região;

- Para a transferência de carga entre qualquer par de nós origem-destino da rede, realizam-se viagens com uma única parada em um terminal de consolidação de carga (seja no Hub Principal ou no mini-hub);

- Os mini-hubs não estão interligados entre eles, mas existe ligação direta entre o Hub Principal e cada mini-hub para movimentar a demanda de carga existente entre eles;

- O mini-hub possui uma área de cobertura de serviço que permite embarques oportunos para fluxos regionais. Ou seja, fixa-se de antemão um raio de cobertura máximo (Rmax), que limita a distância máxima das ligações do mini-hub com os demais locais da sua região de cobertura de serviço;

- Permite que a demanda de carga existente entre cada par de instalações origem-destino seja movimentada através de um caminho de custo mínimo;

- Para os fluxos de carga movimentados através do Hub Principal, que implicam viagens de grandes distâncias de deslocamento, o modelo considera o uso de um veículo de maior capacidade em comparação ao utilizado pelo fluxo regional, via um mini-hub, para, dessa maneira, obter ganhos de escala.

Adotam-se as seguintes hipóteses para a construção do modelo:

- Todos os nós da rede (que representam instalações físicas de uma rede de transporte de encomendas expressa) são potenciais candidatos para tornar-se hubs;

- Define-se, previamente, o número de hubs a serem instalados na rede logística: um único Hub Principal e até p mini-hubs (terminais regionais);

- Para fluxos regionais, o mini-hub tem um raio de cobertura máximo (Rmax), que limita as distâncias dos arcos nó origem $\rightarrow$ mini-hub e mini-hub $\rightarrow$ nó destino;

- Todo par origem-destino pertencente a uma mesma região deve utilizar como ponto de transbordo o mini-hub designado para a sua região;

- Tanto o Hub Principal como os mini-hubs podem ter restrições de capacidade;

- Levando em conta que o Hub Principal tende a agrupar um considerável fluxo de carga e que suas ligações com

Figura 1: Configuração de rede projetada pelo modelo proposto.

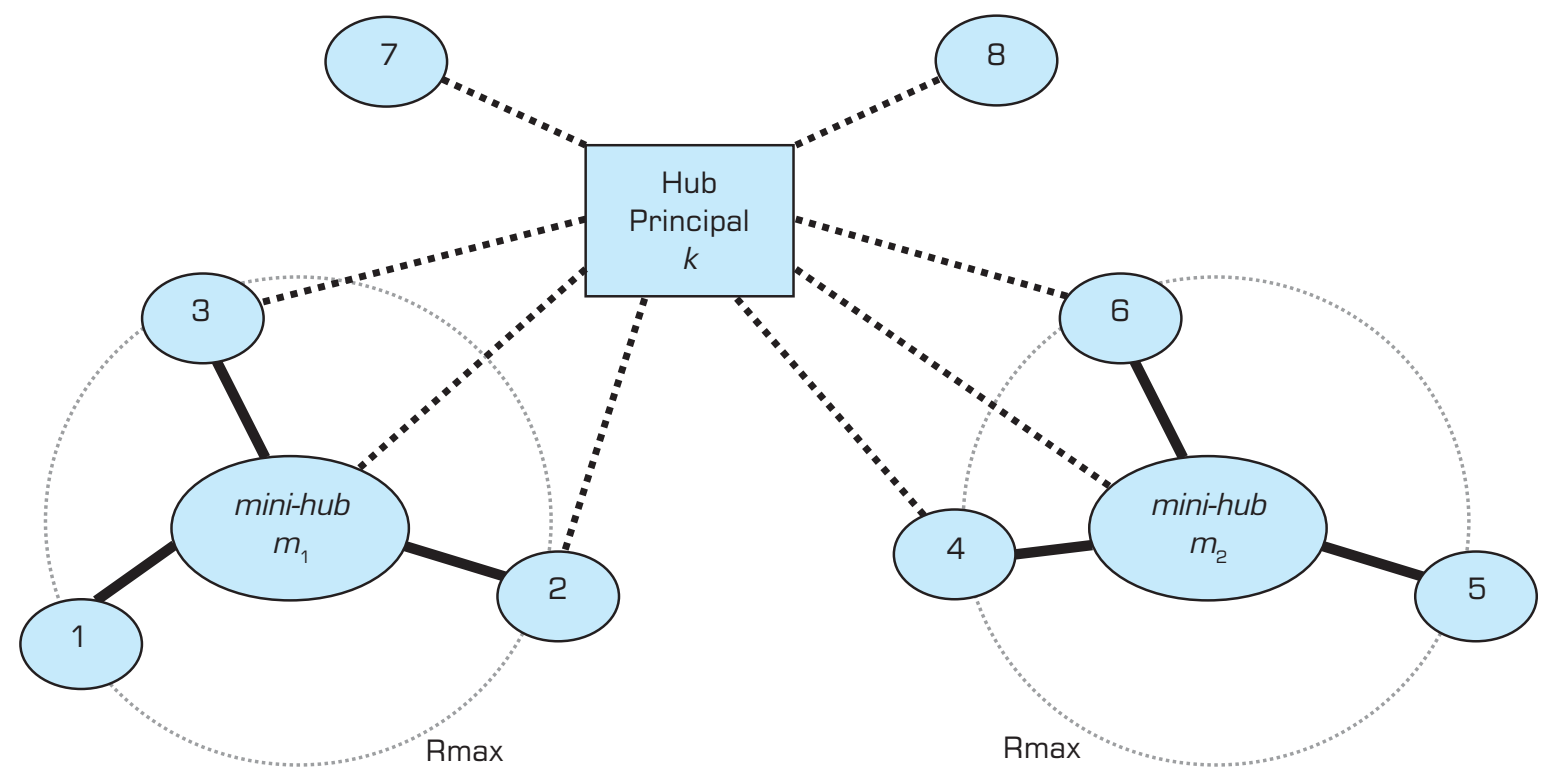


os demais locais implicam viagens de longas distâncias, o modelo considera, para a movimentação da carga por essas ligações, um veículo de maior capacidade de carga em relação ao veículo utilizado para fluxos regionais, conseguindo-se, assim, economias de escala.

\section{MODELO PROPOSTO (MODELO MP)}

A formulação matemática proposta é detalhada por meio de um modelo de programação linear inteira que possui índices, parâmetros e variáveis de decisão abaixo descritos:

\section{Índices:}

$\mathrm{i}, \mathrm{j}$ : para indicar os pontos de origem e destino da carga;

k: $\quad$ para indicar a posição do Hub Principal;

m: para indicar a posição do mini-hub.

\section{Parâmetros:}

$\mathrm{N}$ : indica o número de instalações físicas existentes na rede;

$\mathrm{W}_{\mathrm{ij}}$ : demanda de transporte entre o nó origem i e o nó destino $\mathbf{j}$;

p: número máximo de mini-hubs a serem instalados;

$\mathrm{d}_{\mathrm{im}}$ : distância entre o nó origem i e o mini-hub localizado em $\mathbf{m}$;

$\mathrm{d}_{\mathrm{mj}}$ : distância entre o mini-hub localizado em $\mathbf{m}$ e o nó destino $\mathbf{j}$;

$\mathrm{C}_{\mathrm{ik}}$ : custo unitário de transporte (por unidade de carga) entre o nó origem i e o Hub Principal localizado em k;

$\mathrm{C}_{\mathrm{kj}}$ : custo unitário de transporte (por unidade de carga) entre o Hub Principal localizado em k e o nó destino $\mathbf{j}$;

$\mathrm{c}_{\mathrm{im}}$ : custo unitário de transporte (por unidade de carga) entre o nó origem i e o mini-hub localizado em $\mathbf{m}$;

$\mathrm{c}_{\mathrm{mj}}$ : custo unitário de transporte (por unidade de carga) entre o mini-hub localizado em $\mathbf{m}$ e o destino j;

$\mathrm{F}_{\mathrm{k}}$ : custo fixo de operação do Hub Principal k;

$\mathrm{f}_{\mathrm{m}}$ : custo fixo de operação do mini-hub m;

$\mathrm{R}_{\max }$ : raio máximo de cobertura dos mini-hubs para fluxo de carga regional.

\section{Variáveis de decisão:}

$\mathrm{H}_{\mathrm{k}}=1$, se o Hub Principal é instalado no local k, e igual a 0 , em caso contrário;

$\mathrm{X}_{\mathrm{m}}=1$, se a posição $\mathbf{m}$ é um mini-hub, e igual a 0 , em caso contrário;

$\mathrm{Y}_{\mathrm{ikj}}=1$, se o Hub Principal localizado na posição k é usado para a movimentação da carga entre $\mathbf{i}$ e $\mathbf{j}$, e igual a 0 , em caso contrário;

$\mathrm{Z}_{\text {imj }}=1$, se o mini-hub localizado na posição $\mathbf{m}$ é usado para a movimentação da carga entre i e j, e igual a 0 , em caso contrário;
$\mathrm{V}_{\mathrm{im}}=1$, se o fluxo de carga do nó i para outros nós da mesma região passa pelo mini-hub localizado em $\mathrm{m}$, e igual a 0 , caso contrário.

O modelo proposto MP é apresentado a seguir:

Minimizar

$$
\begin{aligned}
& \mathrm{CT}=\sum_{i=1}^{N} \sum_{j=1}^{N} \sum_{\mathrm{k}=1}^{N} \mathrm{~W}_{\mathrm{ij}}\left(\mathrm{C}_{\mathrm{ik}}+\mathrm{C}_{\mathrm{kj}}\right) \mathrm{Y}_{\mathrm{ikj}}+\sum_{i=1}^{N} \sum_{j=1}^{N} \sum_{m=1}^{N} \mathrm{~W}_{\mathrm{ij}}\left(\mathrm{c}_{\mathrm{im}}+\mathrm{c}_{\mathrm{mj}}\right) \mathrm{Z}_{\mathrm{imj}} \\
& +\sum_{\mathrm{k}=1}^{N}+\mathrm{F}_{\mathrm{k}} \cdot \mathrm{H}_{\mathrm{k}}+\sum_{\mathrm{m}=1}^{N} \mathrm{f}_{\mathrm{m}} \cdot \mathrm{X}_{\mathrm{m}}
\end{aligned}
$$

Sujeito às seguintes restrições:

$$
\sum_{k=1}^{N} H_{k}=1
$$

$\sum_{m=1}^{N} X_{m} \leq p$

$\mathrm{Y}_{\mathrm{ikj}}-\mathrm{H}_{\mathrm{k}} \leq 0 \quad$ para todos $\mathrm{i}, \mathrm{j}, \mathrm{k}$, tais que $\mathrm{i} \neq \mathrm{j}$

$\mathrm{Z}_{\mathrm{imj}}-\mathrm{X}_{\mathrm{m}} \leq 0 \quad$ para todos $\mathrm{i}, \mathrm{j}, \mathrm{m}$, tais que $\mathrm{i} \neq \mathrm{j}$

$\sum_{k=1}^{N} Y_{i k j}+\sum_{m=1}^{N} Z_{i m j}=1$ para todos $\mathrm{i}, \mathrm{j}$, tais que $\mathrm{i} \neq \mathrm{j}$

$\sum_{m=1 \text { e } \neq i}^{N} \mathrm{~V}_{\mathrm{im}} \leq 1 \quad$ para todo $\mathrm{i}$

$\mathrm{V}_{\mathrm{im}} \leq \mathrm{X}_{\mathrm{m}} \quad$ para todos $\mathrm{i}, \mathrm{m}$, tais que $\mathrm{i} \neq \mathrm{m}$

$\sum_{i=1}^{N} Z_{i m j} \leq N . V_{i m} \quad$ para todos $i, m$, tais que $\mathrm{i} \neq \mathrm{m}$

$\sum_{\mathrm{i}=1}^{N} \mathrm{Z}_{\mathrm{imj}} \leq \mathrm{N} . \mathrm{V}_{\mathrm{jm}}$ para todos $\mathrm{j}, \mathrm{m}$, tais que $\mathrm{j} \neq \mathrm{m}$

$\mathrm{X}_{\mathrm{i}}+\mathrm{V}_{\mathrm{im}} \leq 1 \quad$ para todos $\mathrm{i}, \mathrm{m}$, tais que $\mathrm{i} \neq \mathrm{m}$

$\mathrm{d}_{\mathrm{im}} \cdot \mathrm{Z}_{\mathrm{imj}} \leq \mathrm{R}_{\max } \quad$ para todos $\mathrm{i}, \mathrm{j}, \mathrm{m}$, tais que $\mathrm{i} \neq \mathrm{j}$

$\mathrm{d}_{\mathrm{mj}} \cdot \mathrm{Z}_{\mathrm{imj}} \leq \mathrm{R}_{\max } \quad$ para todos $\mathrm{i}, \mathrm{j}, \mathrm{m}$, tais que $\mathrm{i} \neq \mathrm{j}$

$\mathrm{H}_{\mathrm{k}}, \mathrm{X}_{\mathrm{m}}, \mathrm{Y}_{\mathrm{ijk}}, \mathrm{Z}_{\mathrm{ijm}}, \mathrm{V}_{\mathrm{im}} \in\{0,1\}$

A função objetivo representa o custo total de transporte na rede que deve ser minimizado. O custo total da rede equivale à soma da parcela do custo de transporte da carga a ser movimentada (pelo Hub Principal ou mini-hub) e das parcelas do custo fixo instalação de cada tipo de terminal de consolidação. 
A restrição (2) especifica que a posição do único $H u b$ Principal deve ser selecionada a partir dos $\mathbf{N}$ locais candidatos; a restrição (3) estabelece que poderão ser selecionados até p mini-hubs. Esta restrição constitui uma primeira modificação em relação ao modelo de O'Kelly, que considerava a mesma restrição, mas como uma igualdade. ção operacional da carga manipulada pelo Hub Principal. As duas seguintes variantes foram formuladas com o objetivo de forçar a um aumento na carga manipulada pelos mini-hubs, a fim de evitar uma baixa ocupação dos veículos nas ligações com os hubs, que leve a um aumento no custo unitário do transporte.

- Modelo V1MP: modelo capacitado que especifica um fluxo máximo de carga que chega ao Hub Principal. Evita desenhar uma rede onde possa aparecer um Hub Principal com movimentação de carga excessiva (que possa levar a seu congestionamento) e um conjunto de mini-hubs muito dispersos manipulando uma reduzida quantidade de carga;

- Modelo V2MP: impõe a condi-

A restrição (4) impõe que a instalação do Hub Principal na posição k é condição necessária para que a interação entre os nós i e j seja feita via o Hub Principal; a restrição (5) impõe limitação análoga para o mini-hub localizado em m; a restrição (6) especifica que a demanda de transporte do par (i, j) deverá ser atendida seja através de um Hub Principal ou através de um mini-hub. Até aqui, o modelo mantém as mesmas restrições que o modelo proposto por O'Kelly.

A seguir, detalham-se as restrições que foram acrescentadas ao modelo de O'Kelly e que deram forma ao modelo proposto. A restrição (7) assegura que toda instalação i esteja conectada a, no máximo, um único mini-hub $\mathbf{m}$ para o envio da sua carga ao demais locais da sua própria região; a restrição (8) obriga a que todo local i ligado ao mini-hub $m$ encaminhe sua carga às demais instalações da sua região sempre via o mini-hub m; a restrição (9) indica que o local i pode enviar carga até vários destinos $\mathbf{j}$ passando pelo minihub $\mathbf{m}$, somente se o local i está conectado ao mini-hub $\mathbf{m}$; a restrição (10) expressa que somente pode haver movimentação de carga para j a partir de diversos locais i passando pelo mini-hub $\mathbf{m}$, se o local $\mathbf{j}$ está conectado ao mini-hub $\mathbf{m}$; a restrição (11) impede qualquer ligação entre os mini-hubs, já que, se i é mini-hub, ele não pode estar alocado ao mini-hub j; a restrição (12) limita a distância entre o local origem i e o mini-hub m, enquanto a restrição (13) faz o mesmo para a distância entre o local destino j e o mini-hub m.

As restantes restrições impõem que as variáveis de posição $\left(\mathrm{H}_{\mathrm{k},} \mathrm{X}_{\mathrm{m}}\right)$ e as de alocação $\left(\mathrm{Y}_{\mathrm{ijk}}, \mathrm{Z}_{\mathrm{ijm}}\right.$ e $\left.\mathrm{V}_{\mathrm{im}}\right)$ podem assumir somente os valores 0 ou 1 .

\section{EXTENSÕES DO MODELO PROPOSTO}

Foram formuladas três extensões do modelo proposto (modelo MP). A primeira variante considera uma restri- ção de que um mini-hub somente será instalado se a ele foram alocados pelo menos $\mathbf{r}$ locais da rede e, ao mesmo tempo, que todo local conectado a um mini-hub deve interagir com pelo menos q locais da região de cobertura do mini-hub;

- Modelo V3MP: especifica um fluxo mínimo de carga a ser manipulado nos mini-hubs.

\section{ESTUDO DE CASO}

O modelo proposto MP e suas variantes foram aplicados a uma rede de distribuição de 35 nós, pertencente a uma empresa do setor de encomendas expressas do mercado nacional que utiliza exclusivamente o modal rodoviário. Foram escolhidos quatro cenários de análise que consideraram raios de cobertura dos mini-hubs de $300 \mathrm{~km}, 400 \mathrm{~km}, 500 \mathrm{~km} \mathrm{e}$ $600 \mathrm{~km}$, respectivamente.

Para a topologia da rede estudada, as soluções obtidas com o modelo proposto MP permitiram constatar que, à medida que aumenta o raio de cobertura dos mini-hubs, acontece uma lenta redução do custo total da rede, um ligeiro aumento no número de mini-hubs a serem implantados e uma diminuição da carga concentrada pelo $H u b$ Principal. O aumento do número de mini-hubs, com o aumento do raio de cobertura, é um comportamento que deve ser atribuído às características particulares da rede examinada.

De maneira geral, o modelo proposto MP teve soluções consistentes. Verificou-se a tendência de localizar o $\mathrm{Hub}$ Principal na zona de maior densidade de carga da rede ou próximo dela. Além disso, o modelo propõe uma configuração com mini-hubs espalhados de forma homogênea, implantando um mini-hub em cada região do território nacional. 
Também foi possível conferir o atributo do modelo de identificar, para cada par de nós origem-destino, o correspondente caminho de custo mínimo. Isto ficou evidente para os casos em que duas cidades de uma mesma região, embora estejam conectadas a um mini-hub, movimentavam sua demanda de carga via o Hub Principal.

A Figura 2 mostra os custos obtidos pelos modelos formulados para cada cenário. Percebe-se que, quanto maior é o raio de cobertura dos mini-hubs, menor é o custo total da rede. Assim, constata-se que o último cenário, que contempla uma configuração com raio de cobertura dos mini-hubs de $600 \mathrm{~km}$, obteve o menor custo para todos os modelos.

$\mathrm{O}$ aumento gradual do raio de cobertura dos mini-hubs incentiva um crescimento do fluxo regional, levando a implantar terminais regionais que interagem com um maior número de locais, o que provoca um incremento dos volumes de carga manipulados pelos mini-hubs. Ou seja, locais de uma mesma região que, anteriormente, trocavam suas cargas via um Hub Principal, muito distante deles, agora podem utilizar como ponto de transbordo um terminal mais próximo deles, conseguindo uma diminuição do custo de transporte que influi no custo total da rede.

A Tabela 2 apresenta o resumo de todas as soluções obtidas, bem como as economias atingidas no custo total da rede em relação à situação atual.

Verifica-se que todas as soluções propuseram São Paulo como Hub Principal, mas há diversas opções para as posi- ções dos mini-hubs. Assim, para a Região Centro-Oeste, as soluções variaram entre Brasília, Goiânia e Rondonópolis; para a Região Sul, entre Curitiba e Florianópolis; para a Região Sudeste houve um maior número de opções: Belo Horizonte, Juiz de Fora, Uberlândia, Varginha e Rio de Janeiro.

Entre os cenários analisados, o mais adequado é aquela configuração que contempla um raio de cobertura dos minihubs de $600 \mathrm{~km}$, pois houve uma redução de aproximadamente $9,7 \%$ no custo total da rede em relação à estrutura atual da rede de transporte da empresa objeto do estudo de caso. Por esse motivo, 600 km é um raio de cobertura razoável em termos de nível de serviço e tem um grande potencial de implantação, já que além de proporcionar um menor custo, se consegue reduzir neste cenário o congestionamento do Hub Principal, ampliando-se, dessa maneira, a participação dos mini-hubs no atendimento da demanda de transporte da rede. No entanto, a tomada de decisão final precisaria levar em conta aspectos qualitativos do entorno operacional não considerados na modelagem.

Entre os inconvenientes encontrados nos resultados obtidos com o modelo proposto MP, preocupa a presença de uma baixa concentração de carga em alguns mini-hubs e, ao mesmo tempo, o fato de que eles estejam ligados a poucas instalações, o que leva a uma baixa quantidade de carga nas linhas que conectam com os mini-hubs. Outro caso especial que precisa ser aperfeiçoado é o fato de existirem alguns locais que só interagem com o mini-hub conectado a ele.

Figura 2: Custos obtidos pelos modelos formulados para os quatro cenários de análise.

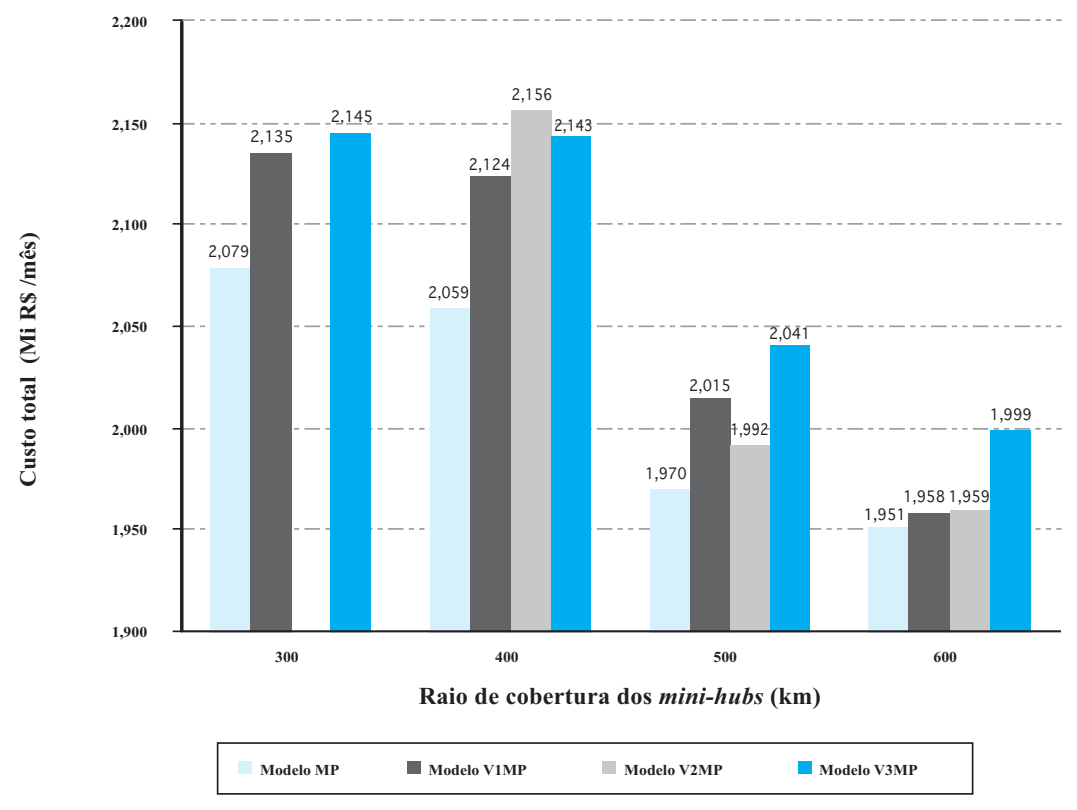


Os resultados computacionais do modelo V1MP permitiram comprovar que a imposição da restrição de capacidade no Hub Principal torna o modelo muito restrito, o que dificulta sua resolução. Assim, em todos os cenários testados, só foram obtidas soluções aproximadas. Além disso, constatou-se que, à medida que se aumenta o raio de cobertura dos mini-hubs, o problema fica menos restrito e diminui a dificuldade de resolvê-lo. rificou que a imposição de um fluxo mínimo de carga nos mini-hubs proporcionava um menor número de mini-hubs a serem implantados, mas se deparava com um aumento na carga concentrada pelo Hub Principal.

Estes efeitos inesperados encontrados com as extensões V2MP e V3MP devem-se, talvez, ao fato de ter examinado uma rede de tamanho médio, conformada somente por 35 nós, e pela presença de duas zonas de considerável densidade

\section{Modelo Irrestrito proposto por O’Kelly
(1998) é a formulação matemática que} proporciona a configuração de rede mais próxima das características desejadas para o serviço de transporte de encomendas expressas.

de carga (sudeste e sul do território nacional) localizadas muito próximas ao Hub Principal indicado pelo modelo.

O modelo proposto e suas extensões foram implementados na linguagem General Algebraic Modelling System (GAMS) e resolvidos por meio do solver CPLEX 7.0. Os testes computacionais foram realizados em um

A estratégia implantada com o modelo V2MP teve pouco efeito, pois se constatou que, embora os mini-hubs chegassem a processar um maior volume de carga, o Hub Principal atingia crescimentos mais consideráveis na quantidade de carga manipulada.

O modelo V3MP teve pouca contribuição, pois se ve- microcomputador equipado com processador Intel Pentium IV operando a 2,26 GHz e $256 \mathrm{MB}$ de memória RAM. Para o caso do modelo proposto (MP), o tempo de processamento foi em média de 16 segundos. Entre as variantes, o modelo V1MP apresentou o maior tempo de processamento, com uma duração média de 554 segundos.

Tabela 2: Resumo das soluções obtidas com os modelos formulados.

\begin{tabular}{|c|c|c|c|c|c|c|c|c|c|c|c|c|c|c|}
\hline \multirow[b]{2}{*}{$\begin{array}{l}\text { Raio de } \\
\text { cobertura } \\
\text { dos mini- } \\
\text { hubs (km) }\end{array}$} & \multirow[b]{2}{*}{ Modelo } & \multirow[b]{2}{*}{$\begin{array}{l}\text { Restriçóes do modelo } \\
\text { capacitado }\end{array}$} & \multirow[b]{2}{*}{$\begin{array}{c}\text { Economias } \\
\text { obtidas } \\
{[\%]}\end{array}$} & \multirow[b]{2}{*}{$\begin{array}{c}\text { Hub Principal } \\
\text { (concentração } \\
\text { carga) }\end{array}$} & \multicolumn{10}{|c|}{ mini-hubs (concentração de carga em \% fluxo totall) } \\
\hline & & & & & 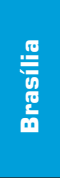 & 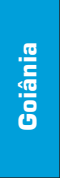 & 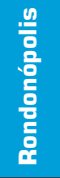 & 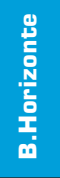 & 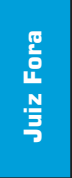 & 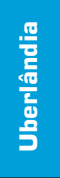 & $\begin{array}{l}\frac{5}{5} \\
\frac{5}{5} \\
\frac{5}{5} \\
\frac{5}{3}\end{array}$ & 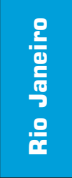 & $\begin{array}{l}\frac{0}{\bar{c}} \\
\frac{\mathrm{g}}{0} \\
\frac{0}{\bar{c}}\end{array}$ & $\begin{array}{l}\frac{8}{2} \\
\frac{2}{5} \\
\frac{5}{3}\end{array}$ \\
\hline \multirow{4}{*}{300} & MP & & $3,7 \%$ & SP (89\%) & $3 \%$ & & & & $5 \%$ & & & & & $3 \%$ \\
\hline & V1MP & fluxo $\max \mathrm{HP}=75 \% \mathrm{ft}$ & & & & & & & & & & & & \\
\hline & V2MP & $r=3 ; q=3$ & $1,1 \%$ & SP (97\%) & & & & & & & & & & $3 \%$ \\
\hline & VЗMP & fluxo $\mathrm{min} \mathrm{mh}=5 \% \mathrm{ft}$ & $0,7 \%$ & SP [100\%) & & & & & & & & & & \\
\hline \multirow{4}{*}{400} & $\mathrm{MP}$ & & $4,7 \%$ & SP (87\%) & & $4 \%$ & & & $5 \%$ & & & & & $4 \%$ \\
\hline & V1MP & fluxo $\max \mathrm{HP}=75 \% \mathrm{ft}$ & $0,1 \%$ & SP (75\%) & & $4 \%$ & & & & & $17 \%$ & & & $4 \%$ \\
\hline & V2MP & $r=3 ; q=3$ & $1,7 \%$ & SP (90\%) & & & & & $5 \%$ & & & & & $4 \%$ \\
\hline & VЗMP & fluxo $\min \mathrm{mh}=5 \% \mathrm{ft}$ & $0,8 \%$ & SP (95\%) & & & & & $5 \%$ & & & & & \\
\hline \multirow{4}{*}{500} & $\mathrm{MP}$ & & $8,8 \%$ & SP (82\%) & & $4 \%$ & $1 \%$ & $6 \%$ & & & & & $7 \%$ & \\
\hline & V1MP & fluxo $\max \mathrm{HP}=75 \% \mathrm{ft}$ & $7,8 \%$ & SP (73\%) & & $4 \%$ & $1 \%$ & & & & & $15 \%$ & $7 \%$ & \\
\hline & V1MP & $r=3 ; q=3$ & $6,7 \%$ & SP (83\%) & & & & $6 \%$ & & $4 \%$ & & & $7 \%$ & \\
\hline & V3MP & fluxo $\min \mathrm{mh}=5 \% \mathrm{ft}$ & $5,5 \%$ & SP (87\%) & & & & $6 \%$ & & & & & $7 \%$ & \\
\hline \multirow{4}{*}{600} & $\mathrm{MP}$ & & $9,7 \%$ & SP (78\%) & & $4 \%$ & $1 \%$ & & & & & $9 \%$ & $7 \%$ & \\
\hline & V1MP & fluxo $\max \mathrm{HP}=75 \% \mathrm{ft}$ & $9,3 \%$ & SP (74\%) & & $4 \%$ & $1 \%$ & & & & & $14 \%$ & $7 \%$ & \\
\hline & V1MP & $r=3 ; q=3$ & $9,3 \%$ & SP (81\%) & & $4 \%$ & & $8 \%$ & & & & & $7 \%$ & \\
\hline & VЗMP & fluxo $\mathrm{min} m \mathrm{mh}=5 \% \mathrm{ft}$ & $7,4 \%$ & SP (80\%) & & & & $8 \%$ & & $5 \%$ & & & $7 \%$ & \\
\hline
\end{tabular}




\section{CONCLUSÕES}

O aprimoramento do modelo de O'Kelly permitiu obter um modelo adequado para resolver o problema de localização-alocação de hubs para uma empresa de transporte de encomendas expressas por possuir os seguintes atributos:

- Garante um menor tempo de transferência da carga entre diferentes instalações logísticas, ao propor uma configuração com roteiros de uma única parada intermediária (seja no Hub Principal ou em um mini-hub);

- Permite identificar, para cada par de interações origemdestino, seu caminho de custo mínimo, possibilitando a escolha do percurso de movimentação da carga, seja via o Hub Principal ou via um mini-hub, tornando-se, assim, um modelo que aborda simultaneamente as fases de localização-alocação-roteirização;

- Permite simplificar as operações de classificação e manuseio da carga nos terminais de consolidação. Assim, ao restringir a passagem da carga por um único hub, reduz-se consideravelmente o número de tarefas de classificação dos pacotes, que implicam um consumo de tempo e despesas de triagem. Isto permitirá implantar uma etapa de pré-classificação na instalação de origem, que separará aqueles pacotes a serem enviados ao Hub Principal ou ao mini-hub, e chegando ali serão submetidos a uma nova classificação de acordo com seu destino final;

- O modelo facilita embarques oportunos que agilizam o fluxo de carga entre instalações logísticas pertencentes a uma mesma região, já que, utilizando como ponto de transbordo o terminal de consolidação mais próximo a eles, permite que a carga faça viagens com menores percursos. Por exemplo, em lugar de impor que a demanda de transporte entre duas instalações físicas muito próximas entre si percorra uma longa trajetória para ir até o Hub Principal, com a configuração proposta pelo modelo, é possível atender a essa demanda com um terminal regional mais próximo das instalações origem e destino.

\section{Artigo recebido em 29/06/2006 Aprovado para publicação em 17/11/2006}

\section{neferências Bibliográficas}

BRYAN, D. L.; O'KELLY, M. E. Hub-andspoke networks in air transportation: an analytical review. Journal of Regional Science, v. 39, n. 2, p. 275-295, 1999.

CAMPBELL, J. F. Integer programming formulations of discrete hub location problems. European Journal of Operational Research, n. 72, p. 387-405, 1994.

DASKIN, M. S; PANAYOTOPOULOS, N. D A Lagrangian Relaxation Approach To Assigning Aircraft To Routes In Hub-AndSpoke Networks. Transportation Science, v. 23, p. 91-99, 1989.

DOBSON, G.; LEDERER, P. J. Airline Scheduling and Routing in a Hub-andSpoke System. Transportation Science, v. 27, p. 281-297, 1993

EBERY, J.; KRISHNAMOORTHY, M ERNEST, A.; BOLAND, N. The capacitated multiple allocation hub location problem: formulations an algorithms. European Journal of Operational Research, n. 120 , p. $614-63,2000$

ERNST, A. T.; KRISHNAMOORTHY, M. Efficient algorithms for the uncapacitated single allocation p-hub median problem. Location Science, v. 4, n. 3, p. 139-154, 1996.

ERNST, A.T. ; KRISHNAMOORTHY, M Exact and heuristic algorithms for the uncapacitated multiple allocation p-hub median problem. European Journal of Operational Research, n. 104 , p. 100 $112,1998$.

ERNST, A. T. ; KRISHNAMOORTHY, M Solution algorithms for the capacitated single allocation hub location problem. Annals of Operational Research, n. 86, p. 141-159, 1999.

FLYNN, J.; RATICK, S. A Multiobjective Hierarchical covering model for the essential Air Services Program. Transportation Science, v. 22, p. 139-147, 1988.

GROVE, P. G. ; O'KELLY, M. E. Hub networks and simulated schedule delay. Papers of the Regional Science Association, v. 59, p. 103-119, 1986.

HALL, R. W. Configuration of an overnight package air network. Transportation Research A, v. 23, p. 139-149, 1989.
JENG, CHAWN-YAW. Routing Strategies for an Idealized Airline Network. Unpublished Ph.D dissertation, University of CaliforniaBerkeley, 1987.

KARA, B. Y.; TANSEL, B. C . On the hub covering problem. Technical report IE-OR 9901, Bilkent University, Department of Industrial Engineerin, 06533, Bilkent, Ankara, Turkey, 1999.

KARA, B. Y. ; TANSEL, B. C. On the single assignment p-hub center problem. European Journal of Operational Research, n. 125, p. $648-655,2000$.

KUBY, M. J. ; GRAY, R. The hub network design problem with stopovers and feeders: The case of Federal Express. Transportation Research A, n. 27, p. 1-12, 1993.

MILLER, H. J. Route 6: Routing algorithm for hub networks. Unpublished manuscript, 1990.

O`KELLY, M. E. A quadratic integer program for the location of interacting hub facilities. European Journal of Operational Research, n. 32, p. 393-404, 1987.
O'KELLY, M. E. On the allocation of a set of nodes to a minihub in a package delivery network. Papers in Regional Science: The Journal of the Regional Science Association International, v. 77, n. 1. p. 77-99, 1998.

O'KELLY, M. E.; BRYAN, D. L. Hub location with economies of scale. Transportation Research B, v. 32, n. 8, p. 605-616, 1998.

O'KELLY, M. E.; LAO, Y. Mode choice in a hub-and-spoke Network: A zero-one linear programming approach. Geographical Analysis, v. 23, p. 283-297, 1991

O'KELLY, M. E.; MILLER, H. J. The hub network design problem: a review and synthesis. Journal of Transport Geography, n. 2, p. 31-40, 1994.

SKORIN-KAPOV, D.; SKORIN-KAPOV, J. O'KELLY, M. E. Tight linear programming relaxations of uncapacitated p-hub median problems. European Journal of Operational Research, n. 94, p. 582-593, 1996. 
- Sobre os autores

Javier Antonio Timaná Alamo

Escola Politécnica da Universidade de São Paulo

Departamento de Engenharia Naval e Oceânica

Doutorando

End.: Av. Prof. Mello Moraes, 2231 - CEP 05508-030 - São Paulo/SP - Brasil

Tel.: (11) 3091-5350 ramal 258

Fax: (11) 3091-5717

E-mail: javier.alamo@poli.usp.br

\section{Marco Antonio Brinati}

Escola Politécnica da Universidade de São Paulo

Departamento de Engenharia Naval e Oceânica

Professor Titular

End.: Av. Prof. Mello Moraes, 2231 - CEP 05508-030 - São Paulo/SP - Brasil

Tel.: (11) 3091-5350 ramal 258

Fax: (11) 3091-5717

E-mail: mabrinat@usp.br 